\title{
Subexponential decay in kinetic Fokker-Planck equation: Weak hypocoercivity
}

\author{
SHULAN HU ${ }^{1}$ and XINYU WANG ${ }^{2}$ \\ ${ }^{1}$ School of Statistics and Mathematics, Zhongnan University of Economics and Law, Wuhan 430073, \\ P. R. China.E-mail: hu_shulan@yahoo.com \\ ${ }^{2}$ School of Mathematics and Statistics, Huazhong University of Science and Technology, Wuhan 430074, \\ P. R. China. E-mail: wang_xin_yu@hust.edu.cn
}

We consider here quantitative convergence to equilibrium for the kinetic Fokker-Planck equation. We present a weak hypocoercivity approach à la Villani, using weak Poincaré inequality, ensuring subexponential convergence to equilibrium in $\mathcal{H}^{1}$ sense or in $L^{2}$ sense.

Keywords: Fokker-Planck equation; hypocoercivity; weak Poincaré inequality

\section{Introduction}

The long-time behavior of the kinetic linear Fokker-Planck equation has been studied for a long time and is by itself a very interesting problem as being one of the simplest hypoelliptic models, noticeably hard to study. From a probabilistic point of view, we are interested in the law of the following degenerate stochastic differential equation on $\mathbb{R}^{n} \times \mathbb{R}^{n}$ :

$$
\left\{\begin{array}{l}
d X_{t}=Y_{t} d t \\
d Y_{t}=\sqrt{2} d B_{t}-\nabla V\left(X_{t}\right) d t-Y_{t} d t .
\end{array}\right.
$$

Let $f(t, x, v)$ be the density of $\left(X_{t}, Y_{t}\right)$. Assume that the equation above admits an invariant measure $d \mu=f_{\infty}(x, v) d x d v$ (with finite or infinite mass), where $f_{\infty}(x, v)$ lies in $C^{2}\left(\mathbb{R}^{n}\right)$ and is positive everywhere. Then the new unknown $h(t, x, v)=f(t, x, v) / f_{\infty}(x, v)$ solves the corresponding partial differential equation:

$$
\frac{\partial h}{\partial t}+v \cdot \nabla_{x} h-\nabla V(x) \cdot \nabla_{v} h=\Delta_{v} h-v \cdot \nabla_{v} h .
$$

Before considering convergence to equilibrium for this model, let us recall some analytical issues about regularity and well-posedness. It was shown by Helffer and Nier [[8], Section 5.2] that (1.1) generates a $C^{\infty}$ regularizing contraction semigroup in $L^{2}(\mu)$ as soon as $V$ lies in $C^{\infty}\left(\mathbb{R}^{n}\right)$, here $d \mu=\frac{e^{-\left[V(x)+\frac{|v|^{2}}{2}\right]}}{Z} d x d v$ with normalization constant $Z$. In [14], Villani used smooth approximation to get the same kind of results under less regular potential $V$. 
Theorem 1.1 (Villani [14]). Let $V \in C^{1}\left(\mathbb{R}^{n}\right)$, lower bounded. Then for any $h_{0} \in L^{2}(\mu)$, equation (1.1) admits a unique distributional solution $h=h(t, x, v) \in C\left(\mathbb{R}_{+} ; \mathcal{D}^{\prime}\left(\mathbb{R}_{x}^{n} \times \mathbb{R}_{v}^{n}\right)\right) \cap$ $L_{\mathrm{loc}}^{\infty}\left(\mathbb{R}_{+} ; L^{2}(\mu)\right) \cap L_{\mathrm{loc}}^{2}\left(\mathbb{R}_{+} ; H_{v}^{1}(\mu)\right)$, such that $h(0, \cdot)=h_{0}$.

If we set $f=\frac{e^{-\left[V(x)+\frac{|v|^{2}}{2}\right]} h}{Z}$, the equation becomes:

$$
\frac{\partial f}{\partial t}+v \cdot \nabla_{x} f-\nabla V(x) \cdot \nabla_{v} f=\Delta_{v} f+\nabla_{v} \cdot(v f) .
$$

$f(t)$ can be interpreted (if it is nonnegative) as a density of particles, or (if it is a probability density) as a law of a random variable in phase space. But the assumptions on the initial datum for (1.2) are more stringent for the well-posedness theorem, see Theorem 7 in [14].

Our main purpose is to study the long time behavior of the solution of this equation. As we all know for symmetric Markov process, a Poincaré inequality for the Dirichlet energy has the same meaning as a Poincaré inequality for the invariant measure $\mu$, which is equivalent to a $L^{2}$ exponential convergence. However due to the degeneracy of the generator associated to the FokkerPlanck equation, a Poincaré inequality related to the Dirichlet energy $\mathcal{E}(f, f)=\int\left|\nabla_{v} f\right|^{2} d \mu$ cannot hold. More generally, for non symmetric Markov process, an exponential convergence in $L^{2}$ sense (not uniform) is not equivalent to a Poincaré inequality for the Dirichlet energy. In this model, coercivity is equivalent to Poincaré inequality or logarithmic Sobolev inequality for the invariant measure $\mu$ previously defined. Under the condition of coercivity, Villani [14] established different kinds of exponential convergence with explicitly computable rates, in $H^{1}$ sense [Theorem 35], in $L^{2}$ sense [Theorem 37], and in the sense of relative entropy [Theorem 39]. Furthermore, Villani [14] (see also [13]) studied hypocoercivity for some dissipative evolution equations which is very similar to problems encountered in the theory of hypoellipticity, and developed a new method for studying convergence problems in a rather systematic and abstract way. It is suggested to refer to the Part I of [14] for a nice presentation of coercivity and hypocoercivity.

Recently, J. Dolbeault, C. Mouhot, and C. Schmeiser [6] used a new approach to prove hypocoercivity for a large class of linear kinetic equations with only one conservation law. They also gave the exponential decay in $L^{2}(\mu)$ for our model.

On the other hand, there are fewer examples about subexponential decay. In [2] Remark 6.7, Bakry, Cattiaux and Guillin gave an example of the subexponential decay by using Lyapunov function (Meyn-Tweedie's method [9-11]), see also [5] for the quantitative coupling approach. This approach relies however on the calculus of the constants of a "small set" for which only (if available) bad evaluations are known. Remark however that no knowledge of the explicit form of the invariant measure is needed.

In this paper, we will study subexponential decay under the weak coercivity condition and the explicit constants of convergence will be given. Here, weak coercivity means weak Poincaré inequality. For the kinetic linear Fokker-Planck equation, a suitable norm is the key to get the convergence rate. We will use the same choice of the norm as [14]. This paper is organized as follows. In Section 2, we present the definitions and some useful results. The weak hypocoercivity and examples will be given in Sections 3 and 4 separately. In the Appendix, proof of Proposition 3.3 is given. 


\section{Framework and useful results}

\subsection{The kinetic Fokker-Planck equation}

Given a nice (at least $C^{1}$, lower bounded) function $V: \mathbb{R}^{n} \rightarrow \mathbb{R}, \int e^{-V(x)} d x<\infty$. For $(x, v) \in$ $\mathbb{R}^{n} \times \mathbb{R}^{n}$, set

$$
\rho_{\infty}(x, v)=\frac{e^{-\left[V(x)+\frac{|v|^{2}}{2}\right]}}{Z}, \quad \mu(d x d v)=\rho_{\infty}(x, v) d x d v,
$$

where $Z$ is chosen for that $\mu$ is a probability measure on $\mathbb{R}^{n} \times \mathbb{R}^{n}$. Define

$$
\begin{aligned}
A_{i} & :=\frac{\partial}{\partial v_{i}}, \quad A:=\nabla_{v}, \quad B:=v \cdot \nabla_{x}-\nabla V(x) \cdot \nabla_{v}, \\
L & :=-\Delta_{v}+v \cdot \nabla_{v}+v \cdot \nabla_{x}-\nabla V(x) \cdot \nabla_{v} .
\end{aligned}
$$

The associated equation is the kinetic Fokker-Planck equation with confinement potential $V$, in the form

$$
\frac{\partial h}{\partial t}+v \cdot \nabla_{x} h-\nabla V(x) \cdot \nabla_{v} h=\Delta_{v} h-v \cdot \nabla_{v} h .
$$

By direct computation in $L^{2}(\mu)$, we can check that

$$
\begin{aligned}
& A_{i}^{*}=-\frac{\partial}{\partial v_{i}}+v_{i}, \quad A^{*} A=\sum_{i=1}^{n} A_{i}^{*} A_{i}=-\Delta_{v}+v \cdot \nabla_{v}, \\
& B^{*}=-B, \quad L=A^{*} A+B .
\end{aligned}
$$

Then the equation takes the form $\partial h / \partial t+L h=0$. By more calculations, we can get

$$
\begin{aligned}
{\left[A_{i}, A_{j}^{*}\right] } & =A_{i} A_{j}^{*}-A_{j}^{*} A_{i}= \begin{cases}1 & i=j, \\
0 & i \neq j,\end{cases} \\
C_{i} & :=\left[A_{i}, B\right]=A_{i} B-B A_{i}=\frac{\partial}{\partial x_{i}}, \quad C:=\left[C_{1}, \ldots, C_{n}\right]=\nabla_{x}, \\
{\left[A_{i}^{*}, C_{j}\right] } & =A_{i}^{*} C_{j}-C_{j} A_{i}^{*}=0, \quad\left[A_{i}, C_{j}\right]=A_{i} C_{j}-C_{j} A_{i}=0, \\
{\left[B, C_{i}\right] } & =B C_{i}-C_{i} B=\left(v \cdot \nabla_{x}-\nabla V \cdot \nabla_{v}\right) \frac{\partial}{\partial x_{i}}-\frac{\partial}{\partial x_{i}}\left(v \cdot \nabla_{x}-\nabla V \cdot \nabla_{v}\right) \\
& =\sum_{j=i}^{n} \frac{\partial^{2} V}{\partial x_{i} \partial x_{j}} \cdot \frac{\partial}{\partial v_{j}}, \\
{[A, C] } & =\left[A^{*}, C\right]=0, \quad\left[A, A^{*}\right]=I, \quad[B, C]=\nabla^{2} V(x) \cdot \nabla_{v} .
\end{aligned}
$$




\subsection{Semigroup, weak coercivity}

The unbounded operator $S$ is to be thought as the generator of the semigroup $\left(P_{t}\right)_{t \geq 0}: P_{t}=$ $e^{-t S}$. We assume that the semigroup $(t, h) \rightarrow e^{-t S} h$ is continuous as a function of both $t$ and $h$, satisfying the semigroup property $e^{0 S}=\mathrm{Id}, e^{-(t+s) S}=e^{-t S} e^{-s S}$ for $t, s \geq 0$, and

$$
\forall h \in D(S),\left.\quad \frac{d}{d t}\right|_{t=0+} e^{-t S} h=-S h .
$$

Similar to the definition of coercivity, we define $(\beta, \Phi)$-weak coercivity of operator $S$ as follows, which will be abbreviated to weak coercivity.

Definition 2.1 (Weak coercivity). Let $S$ be an unbounded operator on a Hilbert space $\mathcal{H}$, with kernel $\mathcal{K} . D(S)$ is dense in $\mathcal{H} . \beta(s)$ is a nonnegative and non-increasing function on $(0,+\infty)$, $\beta(s) \downarrow 0$, as $s \uparrow+\infty$. And $\Phi: \mathcal{H} \rightarrow[0, \infty]$ satisfies $\Phi(c h)=c^{2} \Phi(h)$. The operator $S$ is said to be $(\beta, \Phi)$-weak coercive on $\mathcal{K}^{\perp}$, if

$$
\beta(s)\langle h, S h\rangle_{\mathcal{H}} \geq\|h\|_{\mathcal{H}}^{2}-s \Phi(h), \quad \forall s>0, h \in \mathcal{K}^{\perp} \cap D(S) .
$$

Remark 2.2. If $\lim _{s \rightarrow 0} \beta(s)=\lambda<+\infty$, we obtain exactly $\lambda$-coercivity. The (weak) coercivity depends on the scalar product. Most of the time, (weak) coercivity is in the sense of $L^{2}(\mu)$ or the modified product $((\cdot, \cdot))$ which will be introduced later in Section 3. If the Hilbert space $\mathcal{H}$ is $L^{2}(\mu)$, the form of this inequality imitates the weak Poincare inequality for the measure $\mu$. In this paper, $\Phi(h)=\|h\|_{\infty}^{2},\|h\|_{\infty}=\sup |f|$. Sometimes, $\Phi(h)=\operatorname{Osc}(h)^{2}:=(\sup f-\inf f)^{2}$.

As previously recalled, the coercivity is equivalent to " $\left\|e^{-t S} h\right\|_{\mathcal{H}}^{2} \leq e^{-2 \lambda t}\|h\|_{\mathcal{H}}^{2}$ for all $h \in \mathcal{H}$ " ([14], Proposition 9). We have here the following theorem.

Theorem 2.3. Assume that $S$ is $(\beta, \Phi)$-weak coercive on $\mathcal{K}^{\perp}$, then

$$
\left\|e^{-t S} h\right\|_{\mathcal{H}}^{2} \leq \inf _{\substack{s>0 \\ \beta(s)>0}}\left\{s \sup _{r \in[0, t]} \Phi\left(e^{-r t} h\right)+\exp [-2 t / \beta(s)]\|h\|_{\mathcal{H}}^{2}\right\}, \quad t>0, h \in \mathcal{K}^{\perp} .
$$

Consequently, if $\Phi\left(e^{-t S} h\right) \leq \Phi(h)$ for any $t \geq 0$, then

$$
\left\|e^{-t S} h\right\|_{\mathcal{H}}^{2} \leq \xi(t)\left[\Phi(h)+\|h\|_{\mathcal{H}}^{2}\right], \quad t>0, h \in \mathcal{K}^{\perp} .
$$

Where $\xi(t):=\inf \left\{s>0:-\frac{1}{2} \beta(s) \log s \leq t\right\}$ for $t>0$. In particular, $\xi(t) \downarrow 0$ as $t \uparrow \infty$.

Proof. Assume that $h \in \mathcal{K}^{\perp} \cap D(S)$, let $H(t):=\left\|e^{-t S} h\right\|_{\mathcal{H}}^{2}$. By weak coercivity,

$$
H^{\prime}(t)=-2\left\langle e^{-t S} h, S e^{-t S} h\right\rangle \leq-\frac{2}{\beta(s)} H(t)+\frac{2 s}{\beta(s)} \Phi\left(e^{-t S} h\right), \quad t \geq 0, \beta(s)>0 .
$$

By Gronwall's lemma, we can get the results. 
Remark 2.4. It should be noted that the functional $\Phi$ for the subsequent approach is nonincreasing along the flow.

\subsection{Poincaré inequality and weak Poincaré inequality}

As we know, Poincaré inequality means exponential decay for symmetric Markov process (see [2]), Theorem 1.3. In order to describe convergence rates slower than exponential, the weak Poincaré inequality was introduced by M. Rockner and F.-Y. Wang [12]. We refer to [1] for a nice introduction on the subject of functional inequalities.

Definition 2.5. We say that the measure $\mu$ satisfies a Poincaré inequality, if there exists a constant $C_{P}$, such that, for all $f \in C_{b}^{1}$,

$$
\operatorname{Var}_{\mu}(f):=\left\|f-\int f d \mu\right\|_{2}^{2} \leq C_{P} \int|\nabla f|^{2} d \mu .
$$

Definition 2.6. We say that the measure $\mu$ satisfies a weak Poincaré inequality, if for all $s>0$ and $f \in C_{b}^{1}$,

$$
\operatorname{Var}_{\mu}(f) \leq \beta(s) \int|\nabla f|^{2} d \mu+s\|f\|_{\infty}^{2},
$$

here, $\beta(s)$ is a nonnegative and non-increasing function on $(0,+\infty), \beta(s) \downarrow 0$, as $s \uparrow+\infty$.

It has to be noted that for $d \mu=e^{-V} d x$ a probability measure with $V$ locally bounded, there exists $\beta$ such that $\mu$ verifies a weak Poincaré inequality. Thus, our theorems around convergence of the kinetic Fokker-Planck equation will furnish convergence estimates for nearly all the cases left by Villani.

Remark 2.7. Sometimes we consider another form of the weak Poincaré inequality (see [4]):

$$
\operatorname{Var}_{\mu}(f) \leq \beta(s) \int|\nabla f|^{2} d \mu+s \operatorname{Osc}(f)^{2} .
$$

Note that $\operatorname{Osc}(f) \leq 2\|f\|_{\infty}$, the latter is stronger than the former in a sense. Since $\operatorname{Var}_{\mu}(f) \leq$ $\operatorname{Osc}(f)^{2} / 4$, we may set $\beta(s)=0$ for $s \geq 1$ in the former, and $\beta(s)=0$ for $s \geq 1 / 4$ in the latter.

Remark 2.8. Using capacity-measure criterion, Zitt [15], Theorem 29, showed that a weak Poincaré inequality is indeed equivalent to other variants with weaker norms than the $L^{\infty}$ one: if $\phi, \psi$ are two Young functions with $\phi(x)=\psi\left(x^{2}\right)$, let $N_{\phi}$ be the Orlicz norm associated to $\phi$, a measure $\mu$ satisfies a weak Poincaré inequality (2.1) if and only if

$$
\operatorname{Var}_{\mu}(f) \leq \alpha_{\phi}(s) \int|\nabla f|^{2} d \mu+s N_{\phi}\left(f^{2}\right)
$$


with $\alpha_{\phi}(s)=c \beta\left(\hat{\psi}^{-1}(s / 2) / 4\right), c$ an universal constant, and $\hat{\psi}(x)=\left(\left(\psi^{*}\right)^{-1}(1 / x)\right)^{-1}$ where $\psi^{*}$ is the Fenchel-Legendre dual of $\psi$. We may thus for example consider $L^{p}$ norms instead of $L^{\infty}$ norm.

\section{Decay in $\mathcal{H}^{1}$ under weak coercivity of $A^{*} A+C^{*} C$}

For the kinetic Fokker-Planck equation, there is a natural $\mathcal{H}^{1}$-Sobolev norm $\|\cdot\|_{\mathcal{H}^{1}(\mu)}$ :

$$
\|h\|_{\mathcal{H}^{1}(\mu)}^{2}=\|h\|_{2}^{2}+\|A h\|_{2}^{2}+\|C h\|_{2}^{2}=\|h\|_{2}^{2}+\|\nabla h\|_{2}^{2} \text {. }
$$

In Villani [14] Theorem 35, the exponential convergence in $\mathcal{H}^{1}$ sense under coercivity of $A^{*} A+$ $C^{*} C$ in $L^{2}(\mu)$ sense was got by the following theorem.

Theorem 3.1 (Villani [14]). For the kinetic Fokker-Planck equation, assume that $V \in C^{2}\left(\mathbb{R}^{n}\right)$ with $\left|\nabla^{2} V(x)\right| \leq M(1+|\nabla V|)$ for a constant $M \geq 0$ and the measure $\mu$ satisfies the Poincaré inequality. Then there are constants $C \geq 1$ and $\lambda>0$, explicitly computable, such that for all $h \in \mathcal{H}^{1}(\mu), \int h d \mu=0$,

$$
\left\|e^{-t L} h\right\|_{\mathcal{H}^{1}(\mu)} \leq C e^{-\lambda t}\|h\|_{\mathcal{H}^{1}(\mu)} .
$$

In the above theorem, coercivity of $A^{*} A+C^{*} C$ in $L^{2}$ means Poincaré inequality for invariant measure $\mu$. Next, we will consider the convergence under weak coercivity of $A^{*} A+C^{*} C$ in $L^{2}$, which means weak Poincaré inequality. And the condition for potential $V(x):\left|\nabla^{2} V(x)\right| \leq$ $M(1+|\nabla V|)$ will be replaced by $\left|\nabla^{2} V(x)\right| \leq M$.

There is an associated scalar product to the $\mathcal{H}^{1}$-Sobolev norm, which will be denoted by $\langle\cdot, \cdot\rangle_{\mathcal{H}^{1}}$. Furthermore, we define $((\cdot, \cdot))$ :

$$
((h, h))=\|h\|_{2}^{2}+a\|A h\|_{2}^{2}+2 b\langle A h, C h\rangle_{2}+c\|C h\|_{2}^{2} .
$$

Here $c \leq b \leq a$ and $b^{2}<a c$. We can see that $\langle\cdot, \cdot\rangle_{\mathcal{H}^{1}}$ and $((\cdot, \cdot))$ define equivalent norms:

$$
\begin{aligned}
\min (1, a, c)\left(1-\frac{b}{\sqrt{a c}}\right)\|h\|_{\mathcal{H}^{1}}^{2} & \leq((h, h)) \\
& \leq \max (1, a, c)\left(1+\frac{b}{\sqrt{a c}}\right)\|h\|_{\mathcal{H}^{1}}^{2} .
\end{aligned}
$$

By polarization, we can define the scalar product $((\cdot, \cdot))$. So we easily get the following proposition.

Proposition 3.2. For $h \in \mathcal{H}^{1} \cap D(L)$,

$$
-\frac{1}{2} \frac{d}{d t}\left(\left(e^{-t L} h, e^{-t L} h\right)\right)=\left(\left(e^{-t L} h, L e^{-t L} h\right)\right) .
$$


In particular,

$$
-\left.\frac{1}{2} \frac{d}{d t}\right|_{t=0}\left(\left(e^{-t L} h, L e^{-t L} h\right)\right)=((h, L h)) .
$$

The proposition below is the key in the next theorem. A proof is provided in the Appendix. It can be seen as a particular case of a result by Villani, with a simpler and self contained proof, which moreover leads to easily traceable constants.

Proposition 3.3. For the kinetic Fokker-Planck equation, we always assume that $V \in C^{2}\left(\mathbb{R}^{n}\right)$ and $\left|\nabla^{2} V(x)\right| \leq M$ with a constant $M \geq 0$, for all $x \in \mathbb{R}^{n}$. There is a constant $\kappa>0$, such that

$$
((h, L h)) \geq \kappa\left(\|A h\|_{2}^{2}+\|C h\|_{2}^{2}\right)
$$

with $\kappa:=\min \left(\frac{3}{4}+a-b M, b-(a+b+c M)^{2}\right)$. For example, we can choose $c<\frac{1}{M(M+2)^{2}}$, $b=c M, a=c M^{2}$.

Remark 3.4. $\left|\nabla^{2} V(x)\right| \leq M$ is satisfied for all the examples which we will consider later. For instance, for $\alpha>0,0<p<2$, let $\tilde{V}(x)=(n+\alpha) \ln (1+|x|)$ and $\tilde{V}(x)=|x|^{p}$. In order to set $V \in C^{2}\left(\mathbb{R}^{n}\right)$, we have to modify them to make them smooth enough in a very small neighborhood of 0 , but $\left|\nabla^{2} V(x)\right| \leq M$ is still correct for the modified $V(x)$. We will see these examples later.

Remark 3.5. Recall that one may weaken the assumption to $\left|\nabla^{2} V(x)\right| \leq M(1+|\nabla V|$ ) (of course, $\kappa$ is then poorly estimated). It is due to the first part of Theorem 18 and Lemma A.24 in Appendix A.23 in Villani's work [14].

\subsection{Convergence to equilibrium in $\mathcal{H}^{1}$}

It is now clear that weak coercivity of $A^{*} A+C^{*} C$ in $L^{2}(\mu)$ is equivalent to weak Poincare inequality for measure $\mu$. By Proposition 3.3, we can get our main results.

Theorem 3.6. Under the hypotheses of Proposition 3.3 and a weak Poincaré inequality

$$
\operatorname{Var}_{\mu}(f) \leq \beta(s) \int|\nabla f|^{2} d \mu+s\|f\|_{\infty}^{2}, \quad s>0,
$$

for the same constant $\kappa$ as in Proposition 3.3 and for $\int h d \mu=0$ and $t>0$, it holds

$$
\left(\left(e^{-t L} h, L e^{-t L} h\right)\right) \leq \inf _{\substack{s>0 \\ \beta(s)>0}}\left(((h, h)) \exp \{-\lambda(s) t\}+\frac{\kappa s}{\beta(s) \lambda(s)} \sup _{0 \leq t^{\prime} \leq t}\left\|e^{-t^{\prime} L} h\right\|_{\infty}^{2}\right) .
$$

Here,

$$
\lambda(s)=\min \left(\kappa, \frac{\kappa}{\beta(s)}\right)\left(\max (1, a, c)\left(1+\frac{b}{\sqrt{a c}}\right)\right)^{-1}
$$


Furthermore,

$$
\left(\left(e^{-t L} h, L e^{-t L} h\right)\right) \leq \xi(t)\left(((h, h))+\|h\|_{\infty}^{2}\right) .
$$

Here,

$$
\begin{aligned}
\xi(t) & =K \inf _{\substack{s>0 \\
\beta(s)>0}}\left\{\frac{s}{\min (\beta(s), 1)}, t \geq-\frac{1}{\lambda(s)} \log \frac{K s}{\min (\beta(s), 1)}\right\}, \\
K & =\max (1, a, c)\left(1+\frac{b}{\sqrt{a c}}\right) .
\end{aligned}
$$

In particular, $\xi(t) \rightarrow 0$ as $t \rightarrow \infty$.

Proof. By weak Poincaré inequality, we have

$$
\|A h\|_{2}^{2}+\|C h\|_{2}^{2}=\int|\nabla h|^{2} d \mu \geq \frac{1}{\beta(s)}\|h\|_{2}^{2}-\frac{s}{\beta(s)}\|h\|_{\infty}^{2} .
$$

Then,

$$
\begin{aligned}
((h, L h)) \geq & \frac{\kappa}{2}\left(\|A h\|_{2}^{2}+\|C h\|_{2}^{2}\right)+\frac{\kappa}{2}\left(\frac{1}{\beta(s)}\|h\|_{2}^{2}-\frac{s}{\beta(s)}\|h\|_{\infty}^{2}\right) \\
\geq & \kappa^{\prime}\|h\|_{\mathcal{H}^{1}}-\frac{\kappa s}{2 \beta(s)}\|h\|_{\infty}^{2} \quad\left(\kappa^{\prime}=\min \left(\frac{\kappa}{2}, \frac{\kappa}{2 \beta(s)}\right)\right) \\
\geq & \kappa^{\prime \prime}((h, h))-\frac{\kappa s}{2 \beta(s)}\|h\|_{\infty}^{2} \\
& \left(\kappa^{\prime \prime}=\kappa^{\prime}\left(\max (1, a, c)\left(1+\frac{b}{\sqrt{a c}}\right)\right)^{-1}\right) .
\end{aligned}
$$

Let $\lambda(s)=2 \kappa^{\prime \prime}$, we have

$$
\frac{d}{d t}\left(\left(e^{-t L} h, L e^{-t L} h\right)\right) \leq-\lambda(s)\left(\left(e^{-t L} h, L e^{-t L} h\right)\right)+\frac{\kappa s}{\beta(s)}\left\|e^{-t L} h\right\|_{\infty}^{2} .
$$

By Gronwall's lemma, we can finish the first conclusion. Noticing $\left\|e^{-t L} h\right\|_{\infty}^{2} \leq\|h\|_{\infty}^{2}$ and setting $\frac{\kappa s}{\beta(s) \lambda(s)} \leq \exp \{-\lambda(s) t\}$, we get the second part. It is easy to see that $\frac{1}{\lambda(s)}$ is decreasing and $\frac{s}{\min (\beta(s), 1)}$ is increasing, so $\xi(t) \rightarrow 0$ as $t \rightarrow \infty$.

By the equivalence of $\langle\cdot, \cdot\rangle_{\mathcal{H}^{1}}$ and $((\cdot, \cdot))$, we have the following corollary.

Corollary 3.7. With the same notations of theorem above, for $\int h d \mu=0$, we have

$$
\left\|e^{-t L} h\right\|_{\mathcal{H}^{1}}^{2} \leq \frac{1}{\min (1, a, c)\left(1-\frac{b}{\sqrt{a c}}\right)} \xi(t)\left(\max (1, a, c)\left(1+\frac{b}{\sqrt{a c}}\right)\|h\|_{\mathcal{H}^{1}}^{2}+\|h\|_{\infty}^{2}\right)
$$


Remark 3.8. One may find that $L^{\infty}$ control is too strong. Recall Remark 2.8, one may get another version under weaker norms ( $L^{p}$ for $p>2$ or more general Orlicz norms), but with a slower rate. One may also use truncation arguments to do so.

The previous theorem suffers however from a serious drawback. Indeed it is required that the initial condition is in $\mathcal{H}^{1}$. However, it can be improved by the regularization technique.

\subsection{Convergence to equilibrium in $L^{2}$}

From the work of Guillin-Wang [7], we can get the gradient estimate of the solutions below.

Lemma 3.9. Let $h$ be a bounded measurable function, then there exists a constant $d_{0}>0$, for all $0<t_{0}<1$, such that

$$
\left\|\nabla e^{-t_{0} L} h\right\|_{2}^{2} \leq \frac{d_{0}\|h\|_{\infty}^{2}}{t_{0}^{3}} .
$$

By direct computation, we have the following statement.

Theorem 3.10. Let $h$ be a bounded measurable function, then there exists a constant $d_{0}>0$, for $0<t_{0}<1$ and $\int h d \mu=0$, such that

$$
\left\|e^{-\left(t+t_{0}\right) L} h\right\|_{2}^{2} \leq d_{1}\left(K\|h\|_{2}^{2}+d_{2}\|h\|_{\infty}^{2}\right) \xi(t)
$$

here, $d_{1}=\left(\min (1, a, c)\left(1-\frac{b}{\sqrt{a c}}\right)\right)^{-1}, d_{2}=\left(\frac{d_{0} K}{t_{0}^{3}}+1\right), K=\max (1, a, c)\left(1+\frac{b}{\sqrt{a c}}\right)$.

\section{Examples for a weak Poincaré inequality}

Two types of potentials in position space for the probability measure $\frac{1}{Z_{V}} e^{-V(x)} d x$ will be considered separately in this section with $V(x) \in C^{2}\left(\mathbb{R}^{n}\right)$, where $Z_{V}$ is the proper normalizing constant. We will verify that all conditions are satisfied for these $V(x)$, then the weak Poincaré inequality can be set up for the invariant measure $\frac{e^{-\left[V(x)+\frac{|v|^{2}}{2}\right]}}{Z} d x d v$. So we can get the conclusions of Theorem 3.6 and Theorem 3.10.

\subsection{For the generalization of $(1+\alpha) \ln (1+|x|)(\alpha>0)$}

For $n=1$, let $\tilde{V}(x)=(1+\alpha) \ln (1+|x|)$. It was shown by Barthe, Cattiaux and Roberto [3] that $\frac{1}{Z_{\tilde{V}}} e^{-\tilde{V}(x)} d x=d \tilde{m}_{\alpha}(x)=\frac{1}{2} \alpha(1+|x|)^{-1-\alpha} d x$ satisfies the weak Poincaré inequality

$$
\operatorname{Var}_{\tilde{m}_{\alpha}}(f) \leq \tilde{\beta}(s) \int|\nabla f|^{2} d \tilde{m}_{\alpha}+s \operatorname{Osc}(f)^{2}
$$


Here $\tilde{\beta}(s)=\tilde{c}_{\alpha} s^{-2 / \alpha}$ for $0<s<1 / 4$, and $\tilde{\beta}(s)=0$ for $s \geq 1 / 4$. But $\tilde{V}(x)=(1+\alpha) \ln (1+|x|)$ is not smooth at point 0 , so we have to modify it. In the following, we will generalize it to the case $n \geq 1$.

Let

$$
V(x)= \begin{cases}(1+\alpha) \ln (1+|x|), & |x| \geq 1 \\ -\frac{3(1+\alpha)}{32} x^{4}+\frac{7(1+\alpha)}{16} x^{2}+(1+\alpha)\left(\ln 2-\frac{17}{32}\right), & |x|<1\end{cases}
$$

It is easy to see that $V(x)$ is $C^{2}(\mathbb{R})$, bounded below by $(1+\alpha)(\ln 2-17 / 32)>0$, and 0 is the median of the measure $\frac{1}{Z_{V}} e^{-V(x)} d x$. By Corollary 4 in [3], we can verify that the modified measure $d m_{\alpha}(x)=\frac{1}{Z_{V}} e^{-V(x)} d x$ still satisfies the weak Poincare inequality $(\operatorname{Osc}(f)$ replaces $\left.\|f\|_{\infty}\right)$ as the original measure $\tilde{m}_{\alpha}(x)$. As $\tilde{m}_{\alpha}(x), \beta(s)=c_{\alpha} s^{-2 / \alpha}$ for $0<s<1 / 4$ and $\beta(s)=0$ for $s \geq 1 / 4$.

Let $m_{\alpha}^{n}$ be the $\mathrm{n}$-fold product of $m_{\alpha}$, i.e. $m_{\alpha}^{n}(x)=\otimes^{n} m_{\alpha}(x)$. Thanks to the sub-additivity property of the variance, $m_{\alpha}^{n}$ satisfies the weak Poincaré inequality

$$
\operatorname{Var}_{m_{\alpha}^{n}}(f) \leq c_{\alpha}\left(\frac{s}{n}\right)^{-2 / \alpha} \int|\nabla f|^{2} d m_{\alpha}^{n}+s \operatorname{Osc}(f)^{2}, \quad 0<s<1 / 4 .
$$

Since the gaussian measure $d \gamma^{n}(v)=(2 \pi)^{-\frac{n}{2}} e^{-\frac{|v|^{2}}{2}} d v$ satisfies the Poincare inequality with $C_{p}=1$, the product measure $m_{\alpha}^{n} \otimes \gamma^{n}$ satisfies the weak Poincaré inequality:

$$
\operatorname{Var}_{m_{\alpha}^{n} \otimes \gamma^{n}}(f) \leq \beta(s) \int|\nabla f|^{2} d m_{\alpha}^{n} \otimes \gamma^{n}+s\|f\|_{\infty}^{2},
$$

where $\beta(s)=\max \left(\frac{1}{4}, \frac{1}{4} c_{\alpha}\left(\frac{s}{n}\right)^{-2 / \alpha}\right)$ for $0<s<1$, and $\beta(s)=0$ for $s \geq 1$.

In the other hand, it is easy to verify that $\left|\nabla^{2} V(x)\right| \leq M$ for a constant $M \geq 0$. Then the results of convergence in $\mathcal{H}^{1}$ and in $L^{2}$ can be established for this case by the theorems before.

Corollary 4.1. If the potential belongs to the type of $(1+\alpha) \ln (1+|x|)$, there exist constants $K \geq 1, c_{\alpha, n}>0, \kappa>0$, such that for $\int h d \mu=0$,

$$
\left(\left(e^{-t L} h, L e^{-t L} h\right)\right) \leq \xi(t)\left(((h, h))+\|h\|_{\infty}^{2}\right) .
$$

Here,

$$
\xi(t)=K \inf _{\substack{s>0 \\ \beta(s)>0}}\left\{\frac{s}{\min (\beta(s), 1)}, t \geq-\frac{1}{\lambda(s)} \log \frac{K s}{\min (\beta(s), 1)}\right\},
$$

with $\beta(s)=\max \left(\frac{1}{4}, \frac{1}{4} c_{\alpha, n} s^{-2 / \alpha}\right)$ and $\lambda(s)=K^{-1} \min \left(\kappa, \frac{\kappa}{\beta(s)}\right)$. Furthermore, for a fixed $h$ with $\int h d \mu=0$, there exits a constant $A>1$ such that for all $t>t_{0}$,

$$
\left\|e^{-t L} h\right\|_{2} \leq A\|h\|_{\infty}[1+t]^{-\frac{\alpha}{2}} .
$$




\subsection{For the generalization of $|x|^{p}(0<p<1)$}

As the steps above, we first consider $n=1$. Let $\tilde{V}(x)=|x|^{p}, \frac{1}{Z_{\tilde{V}}} e^{-\tilde{V}(x)} d x=d \tilde{v}_{p}(x)=$ $\frac{e^{-|x|^{p}}}{2 \Gamma(1+1 / p)} d x$ satisfies a weak Poincaré inequality

$$
\operatorname{Var}_{\tilde{v}_{p}}(f) \leq \tilde{\beta}(s) \int|\nabla f|^{2} d \tilde{v}_{p}+s \operatorname{Osc}(f)^{2}
$$

Here $\tilde{\beta}(s)=\tilde{c}_{p}(\log (2 / s))^{\frac{2}{p}-2}$ for $0<s<1 / 4$, and $\tilde{\beta}(s)=0$ for $s \geq 1 / 4$, see [3]. But $\tilde{V}(x)=$ $|x|^{p}$ is not smooth at point 0 also. In the following, we will deal with it as before.

Let

$$
V(x)= \begin{cases}|x|^{p}, & |x| \geq 1, \\ \frac{p(p-2)}{8} x^{4}+\frac{p(4-p)}{4} x^{2}+\left(1-\frac{3}{4} p+\frac{1}{8} p^{2}\right), & |x|<1 .\end{cases}
$$

It is easy to see that $V(x)$ is $C^{2}(\mathbb{R})$, bounded below by $1-\frac{3}{4} p+\frac{1}{8} p^{2}>0$, and 0 is the median of the measure $\frac{1}{Z_{V}} e^{-V(x)} d x$. By the Corollary 4 in [3], we can verify that the modified measure $d m_{\alpha}(x)=\frac{1}{Z_{V}} e^{-V(x)} d x$ still satisfies the weak Poincaré inequality $\left(\operatorname{Osc}(f)\right.$ replaces of $\left.\|f\|_{\infty}\right)$ as the original measure $\tilde{v}_{p}(x)$. As $\tilde{v}_{p}(x), \beta(s)=c_{p}(\log (2 / s))^{\frac{2}{p}-2}$ for $0<s<1 / 4$ and $\beta(s)=0$ for $s \geq 1 / 4$.

Let $v_{p}^{n}$ be the $n$-fold product of $v_{p}$, that is, $v_{p}^{n}(x)=\bigotimes^{n} v_{p}(x)$, then $v_{p}^{n}$ satisfies the inequality:

$$
\operatorname{Var}_{v_{p}^{n}}(f) \leq c_{p}(\log (2 n / s))^{\frac{2}{p}-2} \int|\nabla f|^{2} d v_{p}^{n}+s \operatorname{Osc}(f)^{2}, \quad 0<s<1 / 4 .
$$

Similarly, the product measure $v_{p}^{n} \otimes \gamma^{n}$ satisfies the weak Poincaré inequality:

$$
\operatorname{Var}_{v_{p}^{n} \otimes \gamma^{n}}(f) \leq \beta(s) \int|\nabla f|^{2} d v_{p}^{n} \otimes \gamma^{n}+s\|f\|_{\infty}^{2},
$$

where $\beta(s)=\max \left(\frac{1}{4}, \frac{1}{4} c_{p}(\log (2 n / s))^{\frac{2}{p}-2}\right)$ for $0<s<1$, and $\beta(s)=0$ for $s \geq 1$.

At last, it is easy to see $\left|\nabla^{2} V(x)\right| \leq M$ for a constant $M \geq 0$.

Corollary 4.2. If the potential belongs to the type of $|x|^{p}$, for $\int h d \mu=0$, there exist constants $K \geq 1, c_{\alpha, n}>0, \kappa>0$, such that

$$
\left(\left(e^{-t L} h, L e^{-t L} h\right)\right) \leq \xi(t)\left(((h, h))+\|h\|_{\infty}^{2}\right) .
$$

Here,

$$
\xi(t)=K \inf _{\substack{s>0 \\ \beta(s)>0}}\left\{\frac{s}{\min (\beta(s), 1)}, t \geq-\frac{1}{\lambda(s)} \log \frac{K s}{\min (\beta(s), 1)}\right\}
$$


with $\beta(s)=c_{\alpha, n}(\log (1 / s))^{\frac{2}{p}-2}$ and $\lambda(s)=K^{-1} \min \left(\kappa, \frac{\kappa}{\beta(s)}\right)$. Furthermore, for a fixed $h$ with $\int h d \mu=0$, there exists a constant $A>1$ such that for all $t>t_{0}$,

$$
\left\|e^{-t L} h\right\|_{2} \leq A\|h\|_{\infty} e^{-t^{p /(2-p)}} .
$$

\section{Appendix}

\section{Proof of Proposition 3.3}

In this Appendix, we will simplify the calculation in C. Villani [14] and give more explicit estimates. We have defined the norm $((\cdot, \cdot))$ :

$$
((h, h))=\|h\|_{2}^{2}+a\|A h\|_{2}^{2}+2 b\langle A h, C h\rangle_{2}+c\|C h\|_{2}^{2} .
$$

Here $c \leq b \leq a$ and $b^{2}<a c$. By polarization,

$$
((h, L h))=\langle h, L h\rangle+a \underbrace{\langle A h, A L h\rangle}_{\mathrm{I}}+c \underbrace{\langle C h, C L h\rangle}_{\mathrm{III}}+b \underbrace{(\langle A L h, C h\rangle+\langle A h, C L h\rangle)}_{\mathrm{II}} .
$$

By $B^{*}=-B$,

$$
\langle h, L h\rangle=\left\langle h, A^{*} A h\right\rangle+\langle h, B h\rangle=\|A h\|^{2} .
$$

For each of the terms I, II and III, the contributions of $A^{*} A$ and $B$ will be estimated separately, and they will be denoted by $\mathrm{I}_{A}, \mathrm{I}_{B}, \mathrm{II}_{A}, \mathrm{II}_{B}, \mathrm{III}_{A}, \mathrm{III}_{B}$.

First of all,

$$
\begin{aligned}
\mathrm{I}_{B} & =\langle A h, A B h\rangle=\sum_{i} \int\left(A_{i} h\right)\left(A_{i} B h\right) d \mu \\
& =\sum_{i} \int\left(A_{i} h\right)\left(B A_{i} h\right) d \mu+\sum_{i} \int\left(A_{i} h\right)\left(A_{i} B h-B A_{i} h\right) d \mu \\
& =0+\langle A h,[A, B] h\rangle=\langle A h, C h\rangle \geq-\|A h\|\|C h\|,
\end{aligned}
$$

where the antisymmetry of $B$ was used. Then,

$$
\begin{aligned}
\mathrm{I}_{A} & =\left\langle A h, A^{*} A h\right\rangle=\sum_{i j}\left\langle A_{i} h, A_{i} A_{j}^{*} A_{j} h\right\rangle=\sum_{i j}\left(\left\langle A_{i} h, A_{j}^{*} A_{i} A_{j} h\right\rangle+\left\langle A_{i} h,\left[A_{i}, A_{j}^{*}\right] A_{j} h\right\rangle\right) \\
& =\sum_{i j}\left(\left\langle A_{j} A_{i} h, A_{i} A_{j} h\right\rangle+\left\langle A_{i} h,\left[A_{i}, A_{j}^{*}\right] A_{j} h\right\rangle\right) \\
& =\sum_{i j}\left(\left\langle A_{i} A_{j} h, A_{i} A_{j} h\right\rangle+\left\langle A_{i} h,\left[A_{i}, A_{j}^{*}\right] A_{j} h\right\rangle\right) \\
& =\left\|A^{2} h\right\|^{2}+\left\langle A h,\left[A, A^{*}\right] A h\right\rangle=\left\|A^{2} h\right\|^{2}+\|A h\|^{2} .
\end{aligned}
$$


Here we used the equality $\left[A, A^{*}\right]=I$ in Section 2.2.

Next,

$$
\begin{aligned}
\mathrm{II}_{B} & =\langle A B h, C h\rangle+\langle A h, C B h\rangle \\
& =\langle A B h, C h\rangle+\langle A h, B C h\rangle+\langle A h,[C, B] h\rangle \\
& =\langle A B h, C h\rangle-\langle B A h, C h\rangle+\langle A h,[C, B] h\rangle \\
& =\|C h\|^{2}+\langle A h,[C, B] h\rangle \\
& \geq\|C h\|^{2}-\|A h\|\|[C, B] h\| \\
& \geq\|C h\|^{2}-M\|A h\|^{2} .
\end{aligned}
$$

(Here $\|[C, B] h\|=\left\|\nabla^{2} V(x) \cdot A h\right\| \leq M\|A h\|$ was used.)

$$
\begin{aligned}
\mathrm{II}_{A} & =\left\langle A A^{*} A h, C h\right\rangle+\left\langle A h, C A^{*} A h\right\rangle \\
& =\sum_{i j}\left(\left\langle A_{i} A_{j}^{*} A_{j} h, C_{i} h\right\rangle+\left\langle A_{i} h, C_{i} A_{j}^{*} A_{j} h\right\rangle\right) \\
& =\sum_{i j}\left(\left\langle A_{j}^{*} A_{i} A_{j} h, C_{i} h\right\rangle+\left\langle\left[A_{i}, A_{j}^{*}\right] A_{j} h, C_{i} h\right\rangle+\left\langle A_{i} h, A_{j}^{*} C_{i} A_{j} h\right\rangle\right) \\
& =\sum_{i j}\left\langle A_{i} A_{j} h, A_{j} C_{i} h\right\rangle+\sum_{i}\left\langle A_{i}, C_{i} h\right\rangle+\sum_{i j}\left\langle A_{j} A_{i} h, C_{i} A_{j} h\right\rangle \\
& =2\left\langle A^{2} h, C A h\right\rangle+\langle A h, C h\rangle \geq-2\left\|A^{2} h\right\|\|C A h\|-\|A h\|\|C h\| .
\end{aligned}
$$

(Here we used the commutation of $\mathrm{C}$ with both $A$ and $A^{*}$.)

Finally,

$$
\begin{aligned}
\mathrm{III}_{B} & =\langle C h, C B h\rangle=\sum_{i}\left\langle C_{i} h, C_{i} B h\right\rangle=0+\sum_{i}\left\langle C_{i} h,\left[C_{i}, B\right] h\right\rangle \\
& \geq-\|C h\|\|[C, B] h\| \geq-M\|A h\|\|C h\|, \\
\mathrm{III}_{A} & =\left\langle C h, C A^{*} A h\right\rangle=\sum_{i j}\left\langle C_{i} h, C_{i} A_{j}^{*} A_{j} h\right\rangle=\sum_{i j}\left\langle C_{i} h, A_{j}^{*} C_{i} A_{j} h\right\rangle \\
& =\sum_{i j}\left\langle A_{j} C_{i} h, C_{i} A_{j} h\right\rangle=\sum_{i j}\left\langle C_{i} A_{j} h, C_{i} A_{j} h\right\rangle=\|C A h\|^{2} .
\end{aligned}
$$

(Here the commutation of $\mathrm{C}$ with both $A$ and $A^{*}$ was used again.)

On the whole, combined with these estimations,

$$
\begin{aligned}
((h, L h)) \geq & \|A h\|^{2}+a\left(\left\|A^{2} h\right\|^{2}+\|A h\|^{2}-\|A h\|\|C h\|\right) \\
& +b\left(\|C h\|^{2}-M\|A h\|^{2}-2\left\|A^{2} h\right\|\|C A h\|-\|A h\|\|C h\|\right)
\end{aligned}
$$




$$
\begin{aligned}
& +c\left(\|C A h\|^{2}-M\|A h\|\|C h\|\right) \\
\geq & (1+a-b M)\|A h\|^{2}-(a+b+c M)\|A h\|\|C h\|+b\|C h\|^{2} \\
& +a\left\|A^{2} h\right\|^{2}-2 b\left\|A^{2} h\right\|\|C A h\|+c\|C A h\|^{2} .
\end{aligned}
$$

Noting $b^{2}<a c, a\left\|A^{2} h\right\|^{2}-2 b\left\|A^{2} h\right\|\|C A h\|+c\|C A h\|^{2} \geq 0$. Then

$$
((h, L h)) \geq\left(1+a-b M-\frac{1}{4}\right)\|A h\|^{2}+\left(b-(a+b+c M)^{2}\right)\|C h\|^{2} .
$$

It is easy to choose $a, b, c$, such that $\kappa:=\min \left(\frac{3}{4}+a-b M, b-(a+b+c M)^{2}\right)>0$. For example, we can choose $c<\frac{1}{M(M+2)^{2}}, b=c M, a=c M^{2}$.

\section{Acknowledgements}

We are grateful to to Prof. Arnaud Guillin. He offered invaluable assistance, support and guidance. This work is also supported by NNSF of China (Grant No. 11771161) and by NSSF of China (Grant No. 17BTJ034).

\section{References}

[1] Ané, C., Blachère, S., Chafaï, D., Fougères, P., Gentil, I., Malrieu, F., Roberto, C. and Scheffer, G. (2000). Sur les Inégalités de Sobolev Logarithmiques. Panoramas et Synthèses [Panoramas and Syntheses] 10. Paris: Société Mathématique de France. MR1845806

[2] Bakry, D., Cattiaux, P. and Guillin, A. (2008). Rate of convergence for ergodic continuous Markov processes: Lyapunov versus Poincaré. J. Funct. Anal. 254 727-759. MR2381160

[3] Barthe, F., Cattiaux, P. and Roberto, C. (2005). Concentration for independent random variables with heavy tails. Appl. Math. Res. Express. AMRX 2 39-60. MR2173316

[4] Barthe, F., Cattiaux, P. and Roberto, C. (2006). Interpolated inequalities between exponential and Gaussian, Orlicz hypercontractivity and isoperimetry. Rev. Mat. Iberoam. 22 993-1067. MR2320410

[5] Bitseki Penda, S.-V., Guillin, A., Hairer, M. and Wang, X.Y. Quantitative subexponential convergence to equilibrium of Markov processes. In preparation.

[6] Dolbeault, J., Mouhot, C. and Schmeiser, C. (2015). Hypocoercivity for linear kinetic equations conserving mass. Trans. Amer. Math. Soc. 367 3807-3828. MR3324910

[7] Guillin, A. and Wang, F.-Y. (2012). Degenerate Fokker-Planck equations: Bismut formula, gradient estimate and Harnack inequality. J. Differential Equations 253 20-40. MR2917400

[8] Helffer, B. and Nier, F. (2005). Hypoelliptic Estimates and Spectral Theory for Fokker-Planck Operators and Witten Laplacians. Lecture Notes in Math. 1862. Berlin: Springer. MR2130405

[9] Meyn, S.P. and Tweedie, R.L. (1993). Markov Chains and Stochastic Stability. Communications and Control Engineering Series. London: Springer. MR1287609

[10] Meyn, S.P. and Tweedie, R.L. (1993). Stability of Markovian processes. II. Continuous-time processes and sampled chains. Adv. in Appl. Probab. 25 487-517. MR1234294

[11] Meyn, S.P. and Tweedie, R.L. (1993). Stability of Markovian processes. III. Foster-Lyapunov criteria for continuous-time processes. Adv. in Appl. Probab. 25 518-548. MR1234295 
[12] Röckner, M. and Wang, F.-Y. (2001). Weak Poincaré inequalities and $L^{2}$-convergence rates of Markov semigroups. J. Funct. Anal. 185 564-603. MR1856277

[13] Villani, C. (2006). Hypocoercive diffusion operators. In International Congress of Mathematicians. Vol. III 473-498. MR2275692

[14] Villani, C. (2009). Hypocoercivity. Mem. Amer. Math. Soc. 202950. MR2562709

[15] Zitt, P.-A. (2008). Annealing diffusions in a potential function with a slow growth. Stochastic Process. Appl. 118 76-119. MR2376253

Received June 2015 and revised March 2017 